Moreover, the temporal increase in the AF intensity at 565 $\pm 20 \mathrm{~nm}$ wavelength during myogenic differentiation was similar to the AF profile of dissociated cells from arteriosclerotic vessels at this same wavelength. These data suggest that an AF photonic fingerprint of stem cell-derived myogenic progeny in vitro mimics that of vascular cells ex vivo. following IMT.

\section{THE ROLE OF A NOVEL ANTI-ANGIOGENIC PROTEIN, FKBPL, IN ANGIOGENESIS ASSOCIATED WITH CARDIAC DYSFUNCTION}

${ }^{1}$ A Alqudah, ${ }^{1} \mathrm{R}$ McNally, ${ }^{1} \mathrm{~N}$ Todd, ${ }^{1} \mathrm{D}$ J Grieve, ${ }^{2} \mathrm{~T}$ Robson, ${ }^{1} \mathrm{~L}$ McClements. ${ }^{1}$ Centre for Experimental Medicine, Queen's University Belfast, UK; ${ }^{2}$ Molecular and Cellular Therapeutics, Royal College of Surgeons in Ireland, Dublin, Ireland

\subsection{6/heartjn|-2018-SCF.4}

People with diabetes have a five-fold higher incidence of cardiovascular disease, the leading cause of death globally. FKBPL is a novel angiogenesis-related protein, with a critical role in physiological and pathological angiogenesis. A first-in-class clinical FKBPL peptide mimetic, ALM201, has successfully completed clinical trials for treatment of solid tumours. FKBPL haploinsufficient $\left(F k b p l^{ \pm}\right)$mice, have a pro-angiogenic phenotype, accompanied by vascular dysfunction. Vascular dysfunction is associated with CVD and T2D.

In view of these findings, we now investigate a specific role for FKBPL in angiogenesis associated with cardiac dysfunction. In streptozotocin (STZ)-induced diabetic mice (50 mg/kg i.p. for 5 consecutive days), cardiac FKBPL mRNA levels were downregulated at 12 weeks compared to vehicle controls $(\mathrm{p}<0.05, \mathrm{n}=5)$; this was associated with diastolic dysfunction (e.g. mitral valve E/A ratio). Similarly, in an experimental mouse model of myocardial infarction (MI) associated with severe cardiac ischaemia/hypoxia and increased angiogenesis, FKBPL mRNA $(\mathrm{p}<0.05)$ and protein levels $(\mathrm{p}<0.01)$ were downregulated versus sham controls $(\mathrm{n} \geq 3)$. Complementary in vitro studies using human umbilical vein endothelial cells (HUVEC) demonstrated increased migration and differentiation following 24 hour exposure to hypoxia (1\%) when compared to normoxia $(\mathrm{p}<0.01, \mathrm{n}=6)$. In addition, FKBPL protein levels were downregulated following exposure to hypoxia $(\mathrm{p}<0.01, \mathrm{n}=6)$, whilst activation of HIF-1 $\alpha$ in normoxia by 24 hour DMOG treatment led to a two-fold reduction in FKBPL protein levels $(\mathrm{p}<0.01, \mathrm{n}=3)$. Furthermore, HUVEC exposed to high glucose ( $30 \mathrm{mM}$ for 24 hour) demonstrated downregulation of FKBPL compared to osmotic control $(\mathrm{p}<0.05, \mathrm{n}=3)$. Interestingly, fenofibrate $(50 \mu \mathrm{M})$ treatment was able to restore HUVEC levels of FKBPL in hypoxia $(\mathrm{p}<0.01, \mathrm{n}=3)$. In conclusion, FKBPL may serve a key regulatory role in pathological angiogenesis associated with cardiac dysfunction and, as such, could be promising as a novel biomarker and therapeutic target in this disease setting.

\section{HIF-1 $\alpha$ DEPENDENT AND INDEPENDENT REGULATION OF PP2A IN HUMAN AORTIC SMOOTH MUSCLE CELLS UNDER HYPOXIA}

Ismail Elgenaidi, J Paul Spiers. Department of Pharmacology and Therapeutics, Trinity College, Dublin, Ireland

10.1136/heartjnl-2018-SCF.5
Aims Although hypoxia can modulate the phosphoprotein phosphates system, few studies have addressed if this is mediated through HIF. Therefore, we investigated the involvement of hypoxia-induced HIF-1 $\alpha$ on:

- PP2A activity,

- post-translational modification of PP2Ac, and

- abundance of key enzymes involved in post-translational modification of PP2A in HASMC.

Methods and results HASMC and HAEC were cultured in cell type specific media for 24 hour under normoxic or hypoxic conditions $\left(1 \% \mathrm{O}_{2}\right)$ or following exposure to DMOG $(100 \mu \mathrm{M})$. Effects on mRNA expression, phosphatase activity, post-translational modification and involvement of HIF-1 $\alpha$ were assessed using RT-PCR, immunoblotting, an immunoprecipitation activity assay, ELISA and siRNA transfection. Hypoxia and DMOG decreased mRNA expression of HIF- $1 \alpha$ and PPP2CA in HASMC and HAEC without altering cell viability. In HASMC hypoxia decreased phosphatase activity (total and PP2Ac) without affecting PP2Ac abundance, an effect mimicked by DMOG. Interestingly, hypoxia increased the level of phosphorylated and demethylated PP2Ac. The latter was associated with increased and decreased abundance of PME-1 and LCMT-1 respectively. Knockdown of HIF-1 $\alpha$ prevented the hypoxia-mediated decrease in total phosphatase activity and mRNA expression of PPP2CA. However, it did not alter the effect of hypoxia on the abundance of pPP2Ac, DPP2Ac, LCMT-1 or PME-1.

Conclusion In HASMC, hypoxia inhibits PP2A activity through a HIF-1 $\alpha$ dependent mechanism. In addition, PP2Ac undergoes HIF- $1 \alpha$ independent phosphorylation and demethylation during hypoxia in keeping with changes in the abundance of PME-1 and LMCT-1. The post-translational modification of PP2Ac is consistent with altered assembly of the PP2A holoenzyme and inhibition of activity. Together these data indicate a complex interaction between hypoxia and the PP2A system which warrants further study.

\section{THE ROLE OF A NOVEL ANGIOGENESIS RELATED PROTEIN, FKBPL, IN SPIRAL UTERINE ARTERY REMODELLING IMPORTANT FOR THE PATHOGENESIS OF PREECLAMPSIA}

${ }^{1}$ Ross McNally, ${ }^{1}$ Naomi Todd, ${ }^{1}$ Abdelrahim Alqudah, ${ }^{2}$ Tracy Robson, ${ }^{1}$ David Grieve, 'Lana McClements. 'Centre for Experimental Medicine, SMDB, Queen's University Belfast; ${ }^{2}$ Molecular and Cellular Therapeutics, Royal College of Surgeons in Ireland, Dublin, Ireland

\subsection{6/heartjnl-2018-SCF.6}

Introduction Preeclampsia is a complication which occurs in $5 \%-6 \%$ of pregnancies, characterised by high blood pressure and/or other organ dysfunction in the third trimester of pregnancy. Preeclampsia has short-term risks for the mother and child, but is also associated with remote cardiovascular disease and/or type 2 diabetes mellitus in both. The pathogenesis of preeclampsia is unclear but it appears to be attributed to inappropriate remodelling of spiral uterine artery as a result of dysregulated trophoblast function. We investigated the involvement of novel regulator of developmental and pathological angiogenesis, FKBPL, and its role in the pathophysiology of preeclampsia.

Methods Trophoblast cells (HTR8.SV.neo, BeWo and JAR) were exposed to hypoxic $(1 \%)$ or normoxic $(21 \%)$ conditions 